\title{
Added Value of Coherent Copolar Polarimetry at X-Band for Crop-Type Mapping
}

\author{
Mario Busquier, Juan M. Lopez-Sanchez ${ }^{\circledR}$, Senior Member, IEEE, and Damian Bargiel
}

\begin{abstract}
A set of six spotlight TerraSAR-X images acquired at $\mathrm{HH}$ and VV polarizations in 2009 over an agricultural site in Germany are employed to evaluate the potential contribution of polarimetric features derived from this copolar mode to croptype mapping. Results show that the inclusion of the correlation between copolar channels in the set of input features of the classifier consistently improves the classification performance with respect to the use of only backscattering coefficients. An increase around $8 \%-10 \%$ in overall accuracy, depending on the experiment setup, is achieved. Both user and producer accuracies are improved for all crop types, being the most noticeable contribution for barley, oat, and sugar beet. Different sets of input features, as well as classification and evaluation strategies, are tested in order to assess the robustness of this contribution.
\end{abstract}

Index Terms-Agriculture, classification, polarimetry, synthetic aperture radar (SAR).

\section{INTRODUCTION}

$\mathbf{S}$ YNTHETIC aperture radar (SAR) polarimetry is known to provide sensitivity to scene physical properties (dielectric constant and structure), hence being useful for remote sensing in many applications [1], [2]. Depending on the number of polarimetric channels acquired by the SAR sensor, data are classified in a range that goes from single polarimetry (only one channel) to full polarimetry (four channels). A fully polarimetric system measures all the possible combinations of transmitting and receiving polarizations using an orthogonal basis. The most common case corresponds to the linear basis, i.e., horizontal and vertical polarizations, $\mathrm{H}$ and $\mathrm{V}$, hence providing the following channels: $\mathrm{HH}, \mathrm{HV}, \mathrm{VH}$, and $\mathrm{VV}$. $\mathrm{HH}$ and $\mathrm{VV}$ employ the same polarization in transmission and reception, and thus they are known as copolar channels, whereas VH and HV use different polarizations, and therefore they are called crosspolar channels.

The most common observable in SAR is the backscattering coefficient of each channel, which indicates the power scattered by each resolution cell in the direction of the radar. Moreover, when more than one channel is available, the

Manuscript received April 8, 2019; revised June 11, 2019; accepted August 4, 2019. This work was supported in part by the Spanish Ministry of Science, Innovation and Universities, State Research Agency (AEI), and in part by the European Funds for Regional Development (EFRD) under project TEC2017-85244-C2-1-P. (Corresponding author: Juan M. Lopez-Sanchez.)

M. Busquier and J. M. Lopez-Sanchez are with the Institute for Computer Research (IUII), University of Alicante, 03080 Alicante, Spain (e-mail: juanma-lopez@ieee.org).

D. Bargiel is with the Institute of Geodesy, Remote Sensing and Image Analysis Group, Technische Universität Darmstadt, 64289 Darmstadt, Germany (e-mail: bargiel@geod.tu-darmstadt.de).

Color versions of one or more of the figures in this letter are available online at http://ieeexplore.ieee.org.

Digital Object Identifier 10.1109/LGRS.2019.2933738 complex correlation between channels (i.e., coherence and phase difference) can be measured and is therefore available as an additional information source.

TerraSAR-X is a German SAR satellite that provides the two copolar channels in one of its standard dual-pol modes. Data acquired on this mode have been employed in many application fields, demonstrating the added value of their coherent measure [3]. For instance, correlation and phase difference between $\mathrm{HH}$ and $\mathrm{VV}$ have been exploited for snow depth retrieval [4], sea ice classification [5], rice monitoring [6], and so on.

The general principle of crop classification based on time series of spaceborne SAR data has been proofed in many studies [7]-[9]. This is also true for the TerraSAR-X sensor as time series of measured backscatter have been used for the classification of various crop types in different regions [10], [11].

The starting point of this letter is a study conducted in Germany with time series of TerraSAR-X data without consideration of polarimetric features [12]. The objective of this letter is to show the contribution of coherent copolar polarimetry to crop-type classification. With this aim, we first present previous results obtained with the same data set but based only on the use of the backscattering coefficients of the two channels, $\mathrm{HH}$ and VV. Then, we evaluate the addition of the complex correlation between these two channels, in the form of normalized amplitude and phase. Evaluation is carried out in terms of classification accuracies (overall, user and producer) and kappa coefficient. Different strategies for training and testing are also tested to assess their potential influence. Finally, different sets of features derived from the coherent copolar matrices are also tested to analyze their impact on the methodology.

\section{A. Test Site}

\section{Material And Methods}

The study area is situated in Northern Germany close to the city of Hanover $(52.56 \mathrm{~N}, 9.84 \mathrm{E})$. The area is flat and dominated by agricultural land cover. As shown in Fig. 1 and listed in Table I, there is a large amount of reference information retrieved by field surveys (169 fields in total). Grasslands and nine different crop types were identified on the visited fields. The area is characterized by vital crops supported by modern agricultural techniques and fertile soils. Each of these classes has been split randomly into training and testing fields as illustrated in Table I.

\section{B. TerraSAR-X Data}

A total of six images acquired by TerraSAR-X in HighResolution Spotlight Mode were available for the 2009 


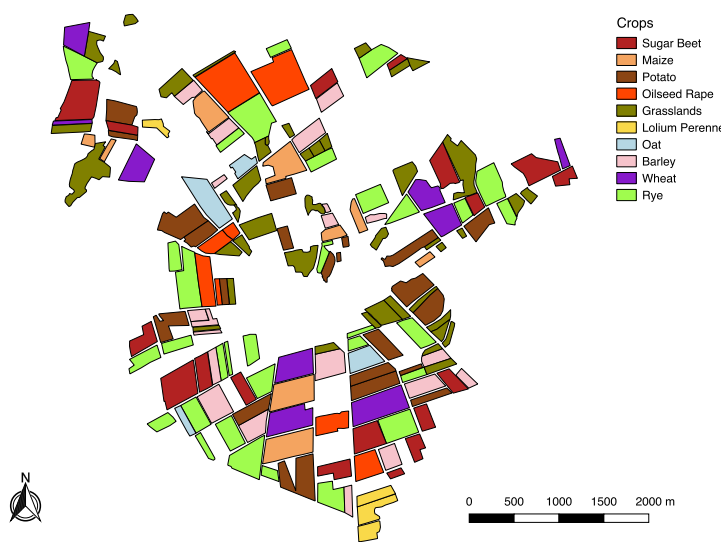

Fig. 1. Crop-type map with all fields available in the ground data.

cultivation period (see Table II). Due to the different phenological condition of crops at different dates, it is known that some dates are better suited than the others for croptype discrimination. However, in this case, we observe the whole cultivation cycle of all crops, with one image per month, approximately, hence minimizing the possible negative effect of missing some key dates.

\section{Data Preprocessing and Classification}

All the single-look complex (SLC) images were preprocessed with the following steps: 1) subset of the region of interest; 2) calibration; 3 ) formation of polarimetric covariance matrices; 4) speckle filtering using a nonlocal polarimetric filter [13]; 5) geocoding; and 6) computation of observables.

The geocoding was carried out to a common Universal Transverse Mercator grid with $2 \mathrm{~m}$ pixel spacing in both coordinates. As for the formation of the covariance matrix, in the copolar dual-pol case, it results in

$$
[C]=\left[\begin{array}{cc}
\left|S_{\mathrm{HH}}\right|^{2} & S_{\mathrm{HH}} \cdot S_{\mathrm{VV}}^{*} \\
S_{\mathrm{VV}} \cdot S_{\mathrm{HH}}^{*} & \left|S_{\mathrm{VV}}\right|^{2}
\end{array}\right]
$$

where $S_{P P}$ denotes the complex amplitude of the image obtained by transmitting and receiving polarization $P=\{\mathrm{H}, \mathrm{V}\}$.

An RGB composite image produced with the data acquired in June is shown in Fig. 2. The image clearly shows different colors for different crop types, which confirms the potential sensitivity of polarimetric data at X-band for crop classification purposes. For instance, short crops dominated by surface-type backscattering appear in blue tones, whereas those in which the double-bounce between stems and ground is the strongest contribution that are shown in reddish colors.

Matrix $[C]$ is Hermitian, so it is fully characterized by four real numbers: the diagonal entries, which are real, and the modulus and phase of the off-diagonal entry, which is complex. Once $[C]$ is properly estimated with the speckle filter, the backscattering coefficients at $\mathrm{HH}$ and $\mathrm{VV}$ correspond directly to the diagonal entries, $C(1,1)$ and $C(2,2)$, respectively. The normalized complex correlation between the two channels is defined as

$$
\rho_{\mathrm{HV}}=\frac{C(1,2)}{\sqrt{C(1,1) \cdot C(2,2)}}
$$

where $\left|\rho_{\mathrm{HV}}\right|$ is the correlation (or coherence) between $\mathrm{HH}$ and $\mathrm{VV}$, and $\arg \left(\rho_{\mathrm{HV}}\right)=\arg (C(1,2))$ is the phase difference between both channels.
These four observables $(\mathrm{HH}, \mathrm{VV}$, correlation, and phase difference) constitute the basic set of features that will be used for classification purposes in Section III. In addition, other features derived from target decomposition and from the expression of the covariance matrix in another basis will be explored.

Classification was carried out with the random forest (RF) classifier [14] using the implementation provided by the scikitlearn package in python. The classifier was run with the default parameters, and they were not changed for the different classification tests described in Section III (i.e., with different input feature sets). Besides these tests, the random split into training and testing fields, and the classification, was run a number of times, always providing very similar results. In an extreme case, the training and testing sets were swapped, and the difference in overall accuracy $(\mathrm{OA})$ was below $1 \%$.

\section{RESUlTS}

\section{A. Initial Classification at Pixel Level}

The initial tests of crop-type mapping coincide with the ones presented in [12] but now employing RF instead of the maximum likelihood classifier (MLC). All pixels of the training set of fields are used for training, and all pixels of the testing set are employed for testing. Results are illustrated in Table III, in which OA, kappa, producer accuracy (PA), and user accuracy (UA) are shown.

First, the two backscattering coefficients, $\mathrm{HH}$ and $\mathrm{VV}$, are used as input features, both alone and in conjunction. The joint use of both channels improves slightly the results with respect to a single channel, increasing by $2 \%$ the OA with respect to the best of both channels (VV). Both UA and PA show minor improvements for some crops, but also some values are worse than one of the two channels. The worst results are obtained for maize, potato, oat, and barley. In the case of maize, an inspection of the temporal evolution of these radar variables shows that they differ importantly among fields, most probably due to their different row orientation. For oat and barley, the problem comes from confusion with other cereals, like wheat and rye, well-reported in the literature, due to similar physical characteristics [15]. The low accuracies for potatoes result probably from the strong influence of rows. Potatoes are planted in characteristic rows of up to $40 \mathrm{~cm}$ height. The direction of these rows to the sensor has a strong influence on the radar backscatter.

In the second place, the correlation between channels is also added as a third input feature of the classifier. From the physical point of view, the correlation between channels is expected to be high (close to 1) when the fields are bare surfaces (i.e., at the beginning and the end of the season), whereas it exhibits lower values when vegetation is present. Table III shows that its inclusion improves notably the performance of the classifier. The OA reaches $76 \%$ (i.e., around $10 \%$ better than without the correlation), and both UA and PA are significantly higher than without the correlation for all crops. For instance, PA increases more than $30 \%$ for oat and barley, reaching values similar to the rest of the crop types. UA is also better than without the correlation, especially for barley, sugar beet, and rye. 
TABLE I

Table of Ground Truth and Data Sets for Training and Testing

\begin{tabular}{|c|c|c|c|c|c|c|}
\hline Crop type & Total number of fields & Total number of pixels & Number of training fields & Number of training pixels & Number of testing fields & Number of testing pixels \\
\hline Sugar beet & 18 & 274,158 & 9 & 119,020 & 15 \\
\hline Maize & 9 & 151,818 & 4 & 76,879 & \\
\hline Potato & 22 & 305,558 & 12 & 158,550 & 5 \\
\hline Oilseed rape & 8 & 198,697 & 4 & 57,528 & 10 \\
\hline Grasslands & 43 & 326,303 & 17 & 111,465 & 4 \\
\hline Lolium Perenne & 4 & 45,279 & 2 & 22,150 & 26 \\
\hline Oat & 4 & 69,058 & 2 & 28,988 & 2 \\
\hline Barley & 20 & 199,585 & 147,008 & 2 \\
\hline Wheat & 9 & 198,438 & 4 & 89,166 & 21,169 \\
\hline Rye & 32 & 447,968 & 14 & 90,858 & 10 \\
\hline
\end{tabular}

TABLE II

LIST OF TERRASAR-X IMAGES

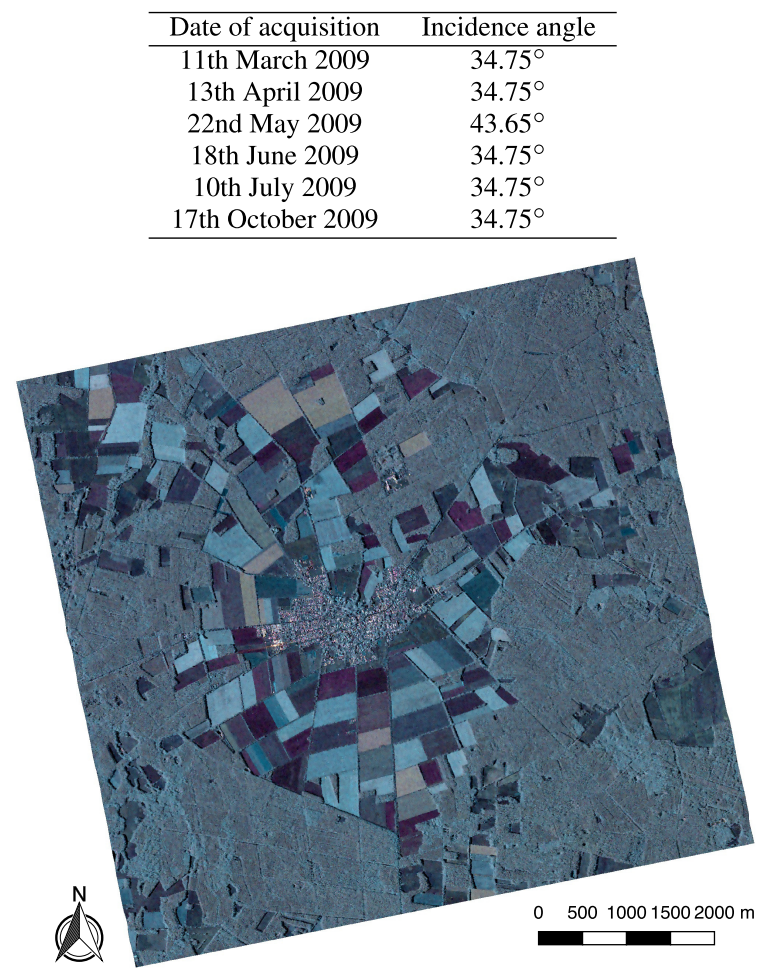

Fig. 2. RGB composite obtained with the image acquired in June 18, 2009. Color coding: red $=\left|S_{\mathrm{HH}}-S_{\mathrm{VV}}\right|^{2}$, green $=\left|S_{\mathrm{VVV}}\right|^{2}$, and blue $=\left|S_{\mathrm{HH}}+S_{\mathrm{VV}}\right|^{2}$.

Finally, the addition of the phase difference between channels in the set of input features does not produce any benefit in the performance of the classifier. This phase is known to be very noisy whenever the correlation is low, whereas it is around zero from bare surfaces. Results provided by the whole set of four features are nominally equal to the ones provided by the previous three features: $\mathrm{HH}, \mathrm{VV}$, and their correlation.

The main conclusion of this experiment is that the availability of a coherent measurement of the copolar channels, not only their powers, is useful for crop-type mapping since its use as an input feature (in form of correlation) produces much better results (around 10\% in OA and 0.1 in kappa). This is the first main finding of this article.

\section{B. Equitable Training Size}

The following test was designed to analyze the potential influence of the unbalanced number of training samples for the different crops, which is a frequent drawback in machine learning methods. Table I shows that the most frequent crops (rye and potato) correspond to a number of samples up to ten times higher than the less frequent ones (lolium and oat). Therefore, in this section, we restricted the number of training samples of all classes to the number of pixels of the class with least pixels (lolium), so all of them were trained in an equitable way. Once trained, the classifier is run over the whole set of testing samples, as in the previous case. The results obtained with this approach are summarized in Table IV, using the same sets of features studied in Section III-A.

Although the final numbers (e.g., OA and kappa) are slightly worse than in Table III, the overall behavior of the classifier is maintained. More importantly, the contribution of the correlation between channels to the performance of the classifier is basically the same, i.e., OA is improved by $8 \%$ with respect to the combined use of the two copolar backscattering coefficients, and PA and UA are notably improved for some crops (e.g., PA increases $25 \%$ for barley and $14 \%$ for potato, while UA increases $28 \%$ for barley and $12 \%$ for grasslands and lolium). As in the previous case, the phase difference between channels does not contribute to improve the results.

\section{Evaluation at Field Level}

Another aspect to be tested is the evaluation of the results at the field level. It is known that in many occasions misclassified pixels are a minority within every field. Therefore, when the objective of the classification is the production of a crop-type map at field level (i.e., one class per field), one can decide the final crop-type at the field level by choosing the mode (i.e., the most frequent class inside each field). The results of this experiment are illustrated in Table V.

In this case, the final results are better than with the previous tests because of the mentioned strategy, since many isolated errors at the pixel level are filtered when evaluating at the field level. It is to be noted that the overall performance (OA and kappa) of the conjunction of $\mathrm{HH}$ and $\mathrm{VV}$ is equal to VV alone, showing only detailed differences for UA and PA at a few crop types. The OA provided by these sets of features is $81 \%$ (kappa $=0.77$ ), which is above the results obtained previously at the pixel level. Nonetheless, the contribution of the correlation between channels is still evident for this scenario, since it provides an OA around $89 \%$ and kappa $=0.86$, hence producing an improvement similar to the one obtained in the previous examples. Consequently, we can state that the contribution of the coherent measurement of the two channels is clear and robust since it does not depend on specific ways to approach the classification problem. 
TABLE III

Results Obtained at Pixel Level With Conventional Classification

\begin{tabular}{|c|c|c|c|c|c|c|c|c|c|c|}
\hline & & \multirow{2}{*}{$\begin{array}{c}\text { Features } \\
\mathrm{OA}(\%)\end{array}$} & $\mathrm{HH}$ & w & \multicolumn{2}{|c|}{$\mathrm{HH}+\mathrm{WV}$} & $\mathrm{HH}+\mathrm{VV}+$ Corr & $\mathrm{HH}+\mathrm{VV}+$ Corr + Phase & \\
\hline & & & 60.43 & 65.63 & \multirow{2}{*}{$\begin{array}{c}67.30 \\
0.62\end{array}$} & \multirow{2}{*}{\multicolumn{2}{|c|}{$\begin{array}{c}76.87 \\
0.73\end{array}$}} & 76.81 & & \\
\hline & & Kappa & 0.54 & 0.60 & & & & 0.73 & & \\
\hline \multicolumn{11}{|c|}{ Producer's Accuracy (\%) } \\
\hline Crop & Sugar beet & Maize & Potato & Oilseed rape & Grasslands & Lolium & Oat & Barley & Wheat & Rye \\
\hline $\mathrm{HH}$ & 64.3 & 17.5 & 53.2 & 95.7 & 80.7 & 59.2 & 14.3 & 19.3 & 60.7 & 63.3 \\
\hline VV & 57.6 & 12.2 & 50.5 & 96.2 & 90.8 & 57.5 & 41.8 & 45.6 & 72.2 & 67.2 \\
\hline$H H+V V$ & 59.6 & 11.8 & 56.5 & 97.3 & 90.0 & 64.2 & 31.2 & 42.8 & 72.8 & 73.0 \\
\hline $\mathrm{HH}+\mathrm{VV}+$ Corr & 74.0 & 18.2 & 70.4 & 98.6 & 91.0 & 60.1 & 64.6 & 72.9 & 81.5 & 78.9 \\
\hline $\mathrm{HH}+\mathrm{VV}+$ Corr + Phase & 74.3 & 16.1 & 70.2 & 98.6 & 90.3 & 61.3 & 63.8 & 72.1 & 80.6 & 80.5 \\
\hline \multicolumn{11}{|c|}{ User's Accuracy (\%) } \\
\hline Crop & Sugar beet & Maize & Potato & Oilseed rape & Grasslands & Lolium & Oat & Barley & Wheat & Rye \\
\hline $\mathrm{HH}$ & 77.4 & 20.9 & 40.5 & 92.6 & 82.4 & 43.5 & 63.4 & 26.8 & 55.3 & 55.7 \\
\hline VV & 70.5 & 16.8 & 36.6 & 92.9 & 85.9 & 69.2 & 85.2 & 56.7 & 60.6 & 66.9 \\
\hline $\mathrm{HH}+\mathrm{VV}$ & 71.9 & 17.4 & 40.4 & 94.0 & 86.2 & 77.8 & 84.3 & 61.2 & 61.7 & 67.6 \\
\hline$H H+V V+$ Corr & 89.3 & 25.0 & 51.0 & 97.8 & 89.0 & 80.4 & 94.1 & 85.2 & 67.3 & 79.8 \\
\hline $\mathrm{HH}+\mathrm{VV}+$ Corr + Phase & 88.5 & 21.9 & 51.8 & 97.7 & 88.8 & 78.2 & 94.3 & 85.3 & 69.1 & 79.1 \\
\hline
\end{tabular}

TABLE IV

Results Obtained at Pixel Level With Same Training Size for All Classes

\begin{tabular}{|c|c|c|c|c|c|c|c|c|c|c|}
\hline & & \multirow{2}{*}{$\begin{array}{c}\text { Features } \\
\text { OA }(\%)\end{array}$} & HH & W & $\mathrm{HH}+\mathrm{VV}$ & \multicolumn{2}{|r|}{$\mathrm{HH}+\mathrm{WV}+$ Corr } & HH + VV + Corr + Phase & & \\
\hline & & & 58.91 & 64.87 & 66.58 & \multicolumn{2}{|r|}{74.79} & 75.40 & & \\
\hline & & Kappa & 0.53 & 0.59 & 0.61 & & 0.71 & 0.71 & & \\
\hline \multicolumn{11}{|c|}{ Producer's Accuracy (\%) } \\
\hline Crop & Sugar beet & Maize & Potato & Oilseed Rape & Grasslands & Lolium & Oat & Barley & Wheat & Rye \\
\hline $\mathrm{HH}$ & 63.6 & 22.4 & 45.2 & 96.7 & 79.8 & 69.9 & 51.1 & 26.5 & 69.4 & 46.5 \\
\hline vv & 57.0 & 15.4 & 46.0 & 97.1 & 89.8 & 64.9 & 63.3 & 54.4 & 79.9 & 54.5 \\
\hline $\mathrm{HH}+\mathrm{VV}$ & 60.9 & 16.6 & 50.7 & 97.9 & 89.6 & 67.1 & 68.9 & 49.8 & 79.5 & 58.7 \\
\hline$H H+V V+$ Corr & 71.1 & 20.3 & 64.7 & 98.7 & 90.9 & 66.1 & 80.6 & 75.3 & 87.3 & 67.5 \\
\hline $\mathrm{HH}+\mathrm{VV}+$ Corr + Phase & 75.4 & 18.3 & 65.5 & 98.7 & 90.2 & 64.9 & 78.0 & 75.7 & 88.1 & 67.6 \\
\hline \multicolumn{11}{|c|}{ User's Accuracy (\%) } \\
\hline Crop & Sugar beet & Maize & Potato & Oilseed Rape & Grasslands & Lolium & Oat & Barley & Wheat & Rye \\
\hline $\mathrm{HH}$ & 76.9 & 20.9 & 40.0 & 87.9 & 81.1 & 24.8 & 67.6 & 27.5 & 53.8 & 61.2 \\
\hline vv & 74.4 & 19.1 & 38.7 & 90.6 & 84.7 & 38.6 & 63.5 & 54.4 & 55.4 & 74.6 \\
\hline $\mathrm{HH}+\mathrm{VV}$ & 76.1 & 18.7 & 42.0 & 91.2 & 85.7 & 52.7 & 75.2 & 53.7 & 54.4 & 74.2 \\
\hline $\mathrm{HH}+\mathrm{VV}+$ Corr & 89.7 & 23.1 & 49.5 & 96.4 & 87.3 & 64.5 & 85.9 & 82.4 & 59.8 & 84.6 \\
\hline $\mathrm{HH}+\mathrm{VV}+$ Corr + Phase & 91.2 & 21.0 & 52.3 & 96.5 & 87.3 & 64.9 & 85.1 & 81.6 & 60.0 & 84.0 \\
\hline
\end{tabular}

TABLE V

Results Obtained With Evaluation at Field LeVEL

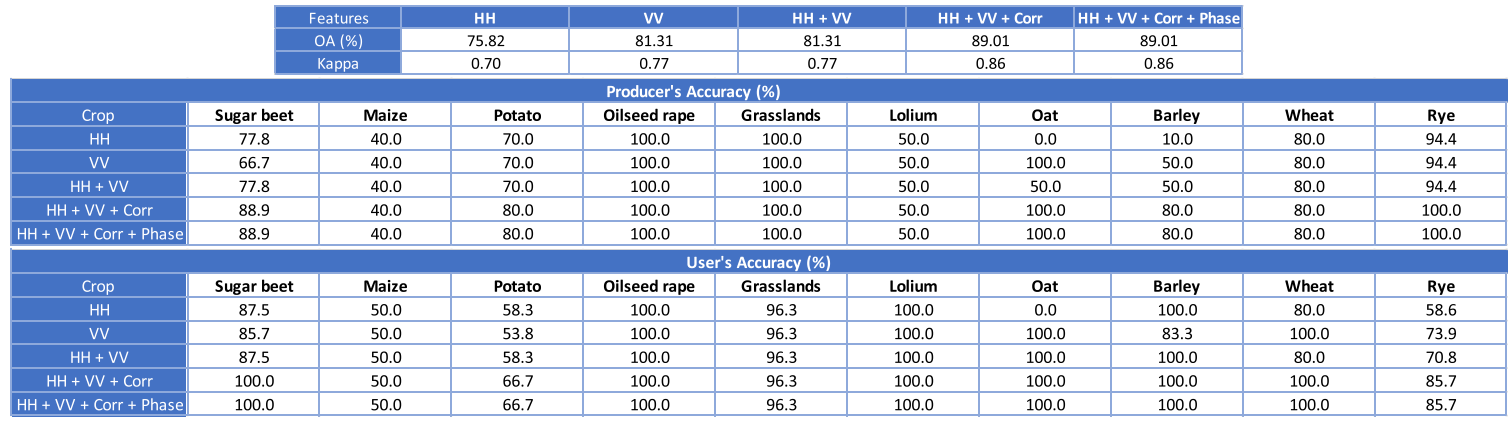

\section{Influence of Input Feature Set}

As a final test, we explore in this section other sets of features, different from the previous ones, but derived from the same data (i.e., copolar covariance matrices). These new features are commonly used in classification with fully polarimetric data, so they are adapted here to the dual-pol copolar case. Their definition is briefly included here.

1) Span: Total backscattered power, obtained as the trace of $[C]$.

2) Eigendecomposition of the Coherency Matrix: Entropy and alpha. The polarization basis can be changed from linear to the Pauli basis, from which the covariance matrix is named the coherency matrix $[T]$. The eigenanalysis of that matrix produces two variables, entropy and alpha, which are widely used in the literature to interpret the type of scattering present in the scene [1], [2].

3) The coherency matrix can be used to obtain the backscattering coefficients of the first two channels of the Pauli basis, i.e., $\left|S_{\mathrm{HH}}+S_{\mathrm{VV}}\right|^{2}$ and $\left|S_{\mathrm{HH}}-S_{\mathrm{VV}}\right|^{2}$, usually recognized as surface and dihedral scattering mechanisms. In addition, the correlation between these two channels can be also derived as it was done for the linear basis in (2).

4) A model-based decomposition based on the FreemanDurden decomposition [16] was adapted to dual-pol copolar data in [6]. The output parameters of this decomposition, hereafter denoted as RVoG, are the power of a volume term (associated with the vegetation), the power of a ground term, and the alpha angle that characterizes the type of scattering of the ground term.

Results obtained with different combinations of these features are summarized in Table VI, in which P1 and P2 refer to the first two Pauli channels and RVoG decomposition denotes the model-based decomposition. Two of the sets provide the same performance of the previous experiments, namely 1) span plus entropy and alpha and 2) model-based decomposition. The use of the span alone (only power information) and entropy and alpha alone (which do not take into account total 
TABLE VI

Results Obtained With Different Sets of InPut Features

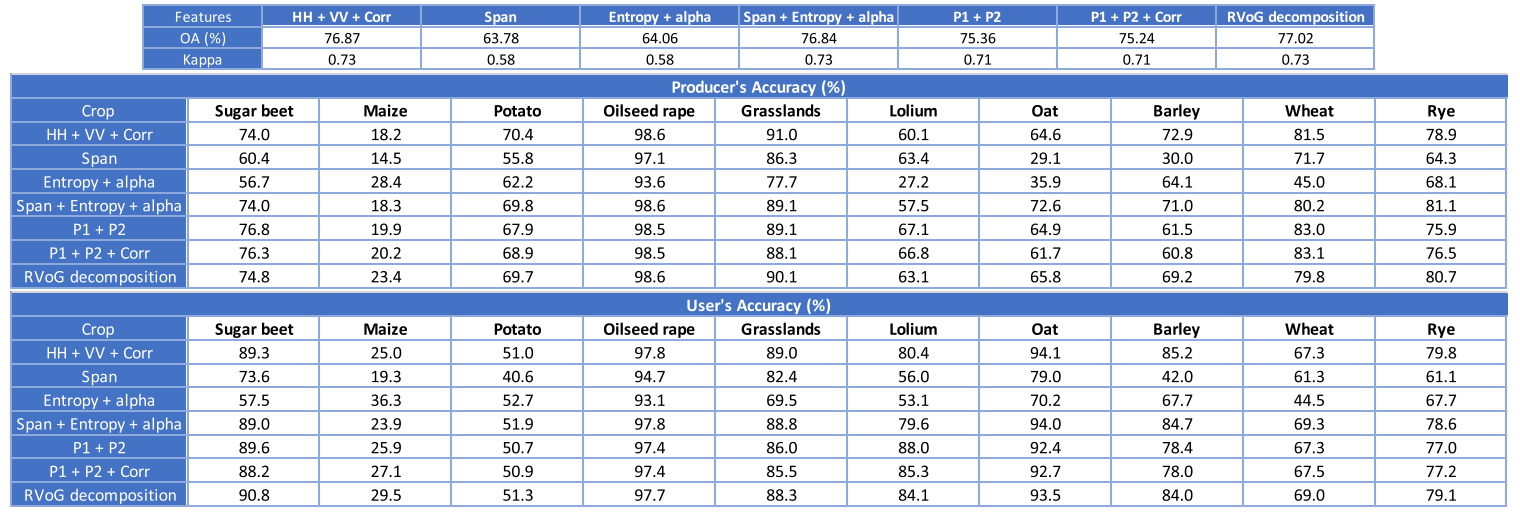

power) provide suboptimal performances, but they complement each other as illustrated in the table.

Regarding the Pauli basis, the results produced with the two backscattering coefficients are almost at the same level of the best results (OA is only $1 \%$ below the best case), which suggests that this selection of polarimetric basis is able to capture most of the scattering diversity present in the data. In fact, the introduction of the correlation between these channels does not improve the results.

Finally, the model-based decomposition provides a marginal improvement with respect to the three features obtained directly from the $[C]$ matrix and in all previous results.

\section{CONCLUSION}

The use of coherent copolar polarimetry at X-band for crop-type mapping has been assessed in this letter with a set of six spotlight images acquired by TerraSAR-X over an agricultural site in Germany. The main novelty of this article is the incorporation of polarimetric features that go beyond the backscattering coefficients of the two copolar channels.

Results show that the inclusion of the correlation between channels in the input set of features improves consistently the classification performance, with an increase around 8\%-10\% in OA, depending on the experiment. Correlation between channels improves the PA and UA of all crop types, being the most noticeable for barley, oat, and sugar beet. Different classification and evaluation strategies have provided similar results, hence demonstrating the robustness of this contribution in crop-type mapping. In addition, the performance of other sets of polarimetric features derived from the copolar mode has been evaluated. In particular, the use of span, entropy, and alpha and the use of outputs of a model-based decomposition provide the same results as the use of the two linear backscattering coefficients and correlation. From all sets of two features, the backscattering coefficients of the first two Pauli channels produce the best results, which are very close to the optimum case.

\section{ACKNOWLEDGMENT}

All synthetic aperture radar (SAR) images have been provided by the German Aerospace Centre (DLR).

\section{REFERENCES}

[1] S. R. Cloude, Polarisation: Applications in Remote Sensing. London, U.K.: Oxford Univ. Press, 2009.

[2] J.-S. Lee and E. Pottier, Polarimetric Radar Imaging: From Basics to Applications. Boca Raton, FL, USA: CRC Press, 2009.

[3] A. Roth et al., "Ten years of experience with scientific TerraSAR-X data utilization," Remote Sens., vol. 10, no. 8, p. 1170, Jul. 2018.

[4] S. Leinss, G. Parrella, and I. Hajnsek, "Snow height determination by polarimetric phase differences in X-band SAR data," IEEE J. Sel. Topics Appl. Earth Observ. Remote Sens., vol. 7, no. 9, pp. 3794-3810, Jun. 2014.

[5] R. Ressel, S. Singha, S. Lehner, A. Rösel, and G. Spreen, "Investigation into different polarimetric features for sea ice classification using X-band synthetic aperture radar," IEEE J. Sel. Topics Appl. Remote Sens., vol. 9, no. 7, pp. 3131-3143, Jul. 2016.

[6] J. M. Lopez-Sanchez, S. R. Cloude, and J. D. Ballester-Berman, "Rice phenology monitoring by means of SAR polarimetry at X-band," IEEE Trans. Geosci. Remote Sens., vol. 50, no. 7, pp. 2695-2709, Jul. 2012

[7] X. Blaes, L. Vanhalle, and P. Defourny, "Efficiency of crop identification based on optical and SAR image time series," Remote Sens. Environ. vol. 96, nos. 3-4, pp. 352-365, Jun. 2005

[8] A. Larrañaga and J. Álvarez-Mozos, "On the added value of quadpol data in a multi-temporal crop classification framework based on RADARSAT-2 imagery," Remote Sens., vol. 8, no. 4, p. 335, Apr. 2016.

[9] H. Skriver, "Crop classification by multitemporal C- and L-band singleand dual-polarization and fully polarimetric SAR," IEEE Trans. Geosci. Remote Sens., vol. 50, no. 6, pp. 2138-2149, Jun. 2012.

[10] R. Sonobe, H. Tani, X. Wang, N. Kobayashi, and H. Shimamura, "Random forest classification of crop type using multi-temporal TerraSAR-X dual-polarimetric data," Remote Sens. Lett., vol. 5, no. 2, pp. 157-164, 2014.

[11] H. McNairn, A. Kross, D. Lapen, R. Caves, and J. Shang, "Early season monitoring of corn and soybeans with TerraSAR$\mathrm{X}$ and RADARSAT-2," Intl. J. Appl. Earth Obs. Geoinf., vol. 28, pp. 252-259, May 2014. [Online]. Available: http://www.sciencedirect. com/science/article/pii/S0303243413001839

[12] D. Bargiel and S. Herrmann, "Multi-temporal land-cover classification of agricultural areas in two European regions with high resolution spotlight TerraSAR-X data," Remote Sens., vol. 3, no. 5, pp. 859-877, Apr. 2011.

[13] C.-A. Deledalle, L. Denis, F. Tupin, A. Reigber, and M. Jäger, "NL-SAR: A unified nonlocal framework for resolution-preserving (Pol)(In)SAR denoising," IEEE Trans. Geosci. Remote Sens., vol. 53, no. 4, pp. 2021-2038, Apr. 2015.

[14] L. Breiman, "Random forests," Mach. Learn., vol. 45, no. 1, pp. 5-32, Oct. 2001

[15] D. Bargiel, "A new method for crop classification combining time series of radar images and crop phenology information," Remote Sens. Environ., vol. 198, pp. 369-383, Sep. 2017.

[16] A. Freeman and S. L. Durden, "A three-component scattering model for polarimetric SAR data," IEEE Trans. Geosci. Remote Sens., vol. 36, no. 3, pp. 963-973, May 1998 